\title{
Detecção do Linfonodo Sentinela em Câncer da Mama: Comparação entre Métodos
}

\author{
Sentinel Lymph Node Detection in Breast Cancer: Comparison between Methods \\ Nilton Leite Xavier, Beatriz Bohrer do Amaral, Bernardo L. Spiro, Maria Janilde de Almeida, \\ Carlos Henrique Menke, Jorge Villanova Biazú, Gilberto Schwartsmann
}

\begin{abstract}
RESUMO
Objetivos: avaliar a eficiência da cintilografia mamária, do detector manual de raios gama (probe) e do corante azul vital para a localização do linfonodo sentinela (LNS), estabelecendo a sensibilidade, o valor preditivo negativo (VPN) e a acurácia do mapeamento do LNS.

Métodos: neste estudo estão incluidas 88 pacientes com câncer de mama atendidas consecutivamente e com axila clinicamente negativa. Foi utilizado o radiofármaco tecnécio ligado ao colóide dextran 500 (Dextran ${ }^{99 m} \mathrm{Tc}$ ), para cintilografia pré-operatória, realizada em 58 pacientes, e detecção transoperatória com o probe em 53 pacientes. Destas, 47 tiveram as contagens de irradiação gama anotadas e também o intervalo de tempo decorrido entre a injeção e a cirurgia. Foram empregados $2 \mathrm{~mL}$ do corante vital Bleu Patente $V$, injetado em torno do tumor, 5 minutos antes da incisão cirúrgica na axila em todos os casos. O LNS foi avaliado por congelação em 77 casos e todos pela coloração por hematoxilina e eosina.

Resultados: a linfocintilografia, realizada em 58 casos, foi útil em 62,1\%, sendo que em nove ocorreu mapeamento de dois linfonodos, e com o probe foram confirmados 45 casos (84,9\%) dos 53 avaliados. Das 47 com anotação de contagens de raios gama e do tempo decorrido da injeção do radiofármaco até a cirurgia, constituíram-se dois grupos para análise, o grupo dia (27 casos) e o grupo outro dia (20 casos), que se mostraram diferentes, com significância estatística, tanto no tempo decorrido quanto nas contagens de raios gama, porém sem interferência nos resultados de detecção do LNS. A associação do probe e do corante azul resultou em sucesso nos 53 casos avaliados, assim como em 32 dos 35 casos em que só o corante foi usado. A média de idade das pacientes foi 58 anos. Quarenta e uma pacientes tiveram linfonodos axilares com metástase e apenas duas com LNS falsamente negativo, dando sensibilidade de 95,3\%, VPN de 95,5\% e acurácia de 97,6.

Conclusões: o mapeamento do LNS é factivel tanto com o uso do tecnécio, aplicado com intervalo variável de 3:00 a 17:00 horas, quanto com o azul vital. Com a associação dos métodos a eficácia foi de 100\%. A confirmação histopatológica, em 97,6\% dos LNS negativos, torna real a possibilidade de evitar a linfadenectomia nestes casos.
\end{abstract}

PALAVRAS-CHAVE: Mama: câncer. Linfadenectomia. Metástase axilar. Linfonodo sentinela.

\section{Introdução}

O estadiamento patológico da axila apresenta valor prognóstico quanto à sobrevida e traz infor-

$\overline{\text { Serviço de Mastologia do Hospital de Clínicas de Porto Alegre }}$ e Departamento de Ginecologia e Obstetrícia da Faculdade de Medicina da UFRGS, Porto Alegre, RS, Brasil.

Correspondência:

Nilton Leite Xavier

Rua João Paetzel, 551 - Bairro: Chácara das Pedras

91330-280 - Porto Alegre - RS

Fone/fax: (51) 3334-0634

E-mail: dr_xavier@terra.com.br mações que influenciam nas decisões terapêuticas adjuvantes imediatas, aumentando a chance de cura clínica; por isso, o estudo que estabeleça o status axilar é fundamental ${ }^{1-4}$.

Coube a Morton et al. ${ }^{5}$ demonstrar a acurácia do mapeamento linfático intraoperatório e a cirurgia seletiva do linfonodo sentinela (LNS), para identificar metástases em linfonodos, em pacientes com melanoma cutâneo primário. Estes autores definiram o LNS como o primeiro linfonodo da rede linfática que drena o tumor ${ }^{1,7} \mathrm{e}$ relataram taxa inferior a $1 \%$ de falso-negativos, 
em mais de 500 casos. Outros trabalhos chegaram aos mesmos resultados, validando o método ${ }^{1,8}$.

Krag et al. ${ }^{1}$ publicaram estudo sobre o LNS em melanoma e logo a seguir os seus resultados em câncer de mama, e concluíram que a radiolocalização e a ressecção seletiva do LNS é possivel e que o LNS parece predizer corretamente o status de todos os demais linfonodos axilares (LN).

Desde os trabalhos de Morton et al. ${ }^{5}$ e Krag et al. ${ }^{1}$ mostrando o valor do estudo do LNS como indicador da primeira metástase, em melanoma, vários estudos foram realizados para o câncer de mama, visando preservar a axila nos casos de LNS negativo (LNS-). O objetivo da dissecção axilar é o estadiamento da doença e a obtenção do controle loco-regional, porém a linfadenectomia traz importante morbidade: linfedema do membro superior ipsilateral, neuropatias, atrofias musculares, ombro dolorido e restrição dos movimentos do braço afetado, além de importantes complicações cirúrgicas como o seroma e a infecção.

O método que estabeleça a localização do LNS, se este representar com erro inferior a $5 \%$, o status axilar, pode trazer o beneficio de oferecer às pacientes uma cirurgia de baixa morbidade, de menor custo e com preservação anatômica.

Três métodos, associados ou não, têm sido propostos para definir a rota preferencial da drenagem linfática do tumor até o LNS: a linfocintilografia mamária, realizada com substância coloidal marcada com o tecnécio $99 \mathrm{~m}$, injetada em torno do tumor e acima do mesmo, obtendo-se a imagem para o diagnóstico 2 horas após injeção do radiofármaco; a utilização, na região axilar, do aparelho de detecção manual de irradiação gama (probe), no pré e transoperatório, usando a mesma substância já injetada para a realização da linfografia, que indica o local do linfonodo que acumulou o radiofármaco ("linfonodo quente") e o uso de um corante azul vital, injetado em torno do tumor e na pele, acima do mesmo, 5 minutos antes de realizar a incisão cirúrgica axilar ${ }^{1,8}$.

Noguchi et al. ${ }^{9}$ referem que há controvérsias a respeito do melhor método de detecção do LNS e, revisando a literatura, concluem que, com a combinação da linfocintilografia pré-operatória, da definição do local através do detector gama-probe e da orientação anatômica obtida pelo corante azul vital, obtém-se as mais altas taxas de identificação do LNS.

Há interesse em estabelecer, para o nosso meio, quais os resultados obtidos com a linfocintilografia mamária e com o detector de irradiação gama, associado ou não ao corante vital usado para linfografia transoperatória. Na prática, as cirurgias nem sempre podem ser realizadas no mesmo dia e, por isso, é importante avaliar se as contagens de irradiação apresentam a mesma média e se os resultados de detecção do LNS são similares.

\section{Pacientes e Métodos}

Noventa pacientes sucessivas, com nódulo mamário, foram prospectivamente avaliadas, no período de 30 de abril 1999 até 30 de junho 2002. Eram oriundas do Serviço de Mastologia do Hospital de Clínicas de Porto Alegre - RS (HCPA), do Hospital Materno-Infantil Presidente Vargas (HMIPV) e de casos pessoais do cirurgião, desta série. Após a aprovação do projeto pelas Comissões de Ética e Pesquisa dos referidos hospitais, foram incluidas as pacientes que preencheram os seguintes critérios: nódulo com câncer, diagnosticado pela clínica e/ou mamografia; nódulo com citopunção ou core biopsy positiva para carcinoma; nódulo com alta suspeição, com dúvida num dos itens acima, e que fosse submetido a biópsia de congelação. Os critérios de exclusão foram: axila clinicamente comprometida, metástase a distância, câncer primário múltiplo, gravidez e quimioterapia prévia.

A média de idade das pacientes foi 58 anos, com limites em 32 e 82 anos. O nódulo, considerando o seu maior diâmetro, apresentou média de $2,5 \mathrm{~cm}$ e limites extremos de 0,8 e 7,0 cm.

Quarenta e nove pacientes foram submetidas a estudo linfocintilográfico usando $2,0 \mathrm{~mL}$ de radiofármaco, constituído de dextran 500 com tecnécio (Dextran $500-{ }^{99 \mathrm{~m}} \mathrm{Tc}$ ) e injetado em torno do tumor da mama, em quatro pontos cardeais e acima do mesmo, em frações divididas de 0,4 $\mathrm{mL}$ em cada um dos cinco pontos. Utilizamos a solução de Dextran $500-{ }^{99 m}$ Tc, distribuída pela Comissão Nacional de Energia Nuclear, São Paulo, por meio do Instituto de Pesquisas Energéticas e Nucleares, cujas partículas variam de 20 a $36 \mathrm{~nm}$, tendo $90 \%$ delas o tamanho de $25 \mathrm{~nm}$, no $\mathrm{pH}$ entre 3 e 4, distribuída em frascos liofilizados, estéreis e apirógenos, em atmosfera de nitrogênio e contendo $100 \mathrm{mg}$ Dextran $500{ }^{99 \mathrm{~m}} \mathrm{Tc}$ e $1,5 \mathrm{mg} \mathrm{SnCl} \mathrm{Sn}_{2} \cdot 2 \mathrm{H}_{2} \mathrm{O}$. Entre uma hora e meia e duas horas após a injeção, obtiveram-se duas imagens, uma ântero-posterior e outra lateral. Para a obtenção da imagem linfocintilográfica utilizou-se a câmara de Anger (gama câmara) marca GE, modelo Millenium MPR com detector retangular e 48 fotomultiplicadores, cristal de 9,0 mm de espessura e variação de energia de 55 a $400 \mathrm{KeV}$. Cinco destas pacientes que receberam a injeção de Dextran ${ }^{99 \mathrm{~m}} \mathrm{Tc}$ foram incluídas na pesquisa usando só o azul vital, por falta do gama probe na data da cirurgia. Outras trinta pacientes usaram apenas o azul vital no transoperatório. Das pacientes que receberam a injeção do Dextran $500-{ }^{99 \mathrm{~m}} \mathrm{Tc}$, apenas 47 tiveram as contagens gama anotadas, sendo que 27 foram operadas no mesmo dia (grupo dia) e 20 o foram no dia seguinte (grupo outro dia).

Todas foram operadas por um dos autores (NLX). Utilizou-se o detector manual Neoprobe ${ }^{\circledR} 1500$ 
para a marcação do ponto de maior contagem de irradiação gama na axila, para a orientação da incisão cirúrgica e procura do LNS. No transoperatório, confirmava-se ou procurava-se encontrar um ponto de contagem gama significativa. Antes da incisão injetava-se $2,0 \mathrm{~mL}$ de azul patente $\mathrm{V}$ (corante vital), com a solução distribuída nos 4 pontos cardeais e sub e intradérmico como quinto ponto, em alíquotas divididas, com seringa de $3,0 \mathrm{~mL}$ e agulha $30 \times 7 \mathrm{~mm}$. O resíduo do azul, seringa, era injetado com $0,2-0,5 \mathrm{~mL}$ de soro fisiológico na pele, acima do nódulo. A mama era massageada por 5 minutos, após o que realizava-se apropriada incisão transversa na axila, procurando o LNS através dos ductos corados e/ou do próprio LNS.

$\mathrm{O}(\mathrm{s})$ LNS foi(foram) seccionado(s) ao meio e uma porção foi usada para citologia por imprint e diagnóstico histológico por congelação, e a outra metade, fixada e processada em bloco de parafina para seções permanentes por hematoxilina e eosina (HE). As lâminas foram avaliadas pelos patologistas do Serviço de Patologia do HCPA e por patologista do convênio com o HMIPV. O tecido removido foi examinado pelas técnicas habituais, avaliando-se o número de linfonodos, a existência de metástase e o diâmetro do tumor.

O valor do mapeamento do LNS foi calculado usando-se a freqüência do sucesso ou da falha do procedimento, seja da cintilografia mamária, da detecção pelo gama probe só ou associado com o corante azul ou do uso apenas do corante vital.

As pacientes, para avaliação da distribuição e estudo da freqüência da ocorrência de LNS com metástase (LNS+) e ou análise bivariada pelo $\chi^{2}$ de Pearson, foram categorizadas em dois grupos quanto à idade: (até 50 anos (IDa)) e maior do que 50 anos (IDb)) e quanto ao tamanho do nódulo (até $2,0 \mathrm{~cm}$ (TAMa)) e maior do que 2,0 cm (TAMb)).

Os dois grupos já referidos, grupo dia e grupo outro dia, tiveram tanto as contagens gama quanto o tempo entre a injeção do tecnécio e a cirurgia avaliados estatisticamente pela ANOVA. A taxa de sucesso do LNS entre os grupos foi avaliada pela estatística do $\chi^{2}$.

A inferência estatística da probabilidade de envolvimento de linfonodo foi baseada na distribuição binomial aplicada para pares de observação; isto é, pela hipótese de nulidade, a metástase pode ocorrer igualmente em LNS e em não-LNS. Para testar esta hipótese escolheu-se o teste de McNemar.

A sensibilidade e outros valores preditivos foram calculados pelo número de pacientes em que as características histológicas dos LNS refletiam as dos demais linfonodos axilares. A unidade de análise foi o número de pacientes e não o número de linfonodos removidos. Os intervalos de confiança dos valores preditivos foram baseados no teste exato de Fisher. O valor de $\mathrm{p}$ foi considerado com significado estatístico quando $<0,05$.

\section{Resultados}

Tivemos duas perdas entre as pacientes que usaram radioisótopo. Uma por erro de técnica, com injeções de tecnécio num local e azul vital distante $2,0 \mathrm{~cm}$ e, na axila, não ocorreu concordância entre linfonodo com irradiação e linfonodo azul. Isto ocorreu porque o hematoma e a dor resultantes da citopunção dificultaram a injeção do radiocolóide, que é realizada sem anestesia. As injeções do radiofármaco e do corante Bleu Patente $\mathrm{V}$ Sodique a 2,5\% foram realizadas apenas por dois dos autores (NLX e BBA) e a concordância do local das duas injeções sempre foi avaliada. Outra paciente foi excluída porque no transoperatório notaram-se dois tumores, distantes de $3,0 \mathrm{~cm}$ entre si. O tumor menor não tinha diagnóstico prévio. Três pacientes avaliadas com o azul vital, não estão incluídas na avaliação anatomopatológica, porque o mapeamento do LNS não foi possível; por isto a avaliação se limitará a 85 casos.

Com o uso do azul vital, três casos não tiveram LNS detectados, ficando o sucesso em 32 casos, ou seja, 91,4\%. Num dos casos de falha procurou-se o LNS por meio do retalho da mastectomia e o ducto linfático teve o seu trajeto seccionado longe da axila e nos outros dois casos, com abordagem axilar, não se observaram os 5 minutos de espera, antes da incisão cirúrgica, nem se realizou a massagem da área peritumoral. Os resultados dos métodos de detecção estão na Tabela 1.

Nesta série, as pacientes com idade menor que 50 anos foram 28 , sendo que $57 \%$ apresentaram metástase no LNS. As $57 \mathrm{com}$ mais de 50 anos tiveram metástase no LNS em 43\%, com $\mathrm{p}=0,249$. $\mathrm{Na}$ caracterização quanto ao diâmetro tumoral, nas 44 pacientes com TAMa $(\mathrm{T} \leq 2 \mathrm{~cm})$ a incidência de LNS com metástase foi $38 \%$, ao passo que em 41 pacientes com TAMb $(\mathrm{T}>2 \mathrm{~cm})$ a porcentagem de metástase axilar foi $58 \%$, com $\mathrm{p}=0,066$.

Quanto ao número de LNS isolados, obtivemos uma média de 2,4 e mediana de 2,0 , com extremos de 1 e 5 . O número médio de linfonodos dissecados, incluindo o LNS, foi de de 20,1, com mediana de 20 e extremos de 10 e 46.

Tabela 1 - Comparação entre as técnicas para mapeamento linfático para identificação do linfonodo sentinela em 88 pacientes.

n Identificação do LNS (\%)

Técnicas pré-operatórias*

Linfocintilografia 58

Radioisótopo com probe

Técnicas transoperatórias

Radioisótopo + corante $53 \quad 53(100)$

Apenas corante $\quad 35 \quad 32(91,4)$

* Foram realizadas em 58 pacientes e o probe foi usado em apenas 53 destas. 
Na Tabela 2 estão as extremos dos valores do tempo decorrido da injeção do radiofármaco até a cirurgia, e dos valores das contagens obtidas com o probe, considerando os dois grupos já referidos. Os grupos dia e outro dia mostraram pela estatística da ANOVA, uma diferença com $F$ de 87,93 para as contagens, e de 670,29 para o tempo (significativos, com $\mathrm{p}<0,001)$. A contagem média de irradiação gama, no grupo do dia, foi de 5853, e a média, no grupo outro dia, foi de 2719 , com diferença estatisticamente significativa e $\mathrm{p}<0,001$.

Tabela 2 - Resultados do grupo dia e grupo outro dia na detecção do linfonodo sentinela.

\begin{tabular}{|c|c|c|c|c|}
\hline Variáveis & Limites & $\mathrm{n}$ & $\%$ LNS+ & Valor de $p \#$ \\
\hline \multicolumn{5}{|l|}{ Tempo de injeção } \\
\hline \multicolumn{5}{|c|}{ até operação: horas } \\
\hline Grupo dia & $3-9$ & 27 & 44 & \\
\hline Grupo outro dia & $14,50-17$ & 20 & 40 & 0,760 \\
\hline \multicolumn{5}{|c|}{$\begin{array}{l}\text { Contagens de irradiação } \\
\text { gama/min. }\end{array}$} \\
\hline Grupo dia & 850-20000 & 27 & 44 & \\
\hline Grupo outro dia & $80-9800$ & 20 & 40 & 0,760 \\
\hline
\end{tabular}

Nas 77 pacientes com avaliação histológica intraoperatória, esta foi positiva em $35,0 \%$ e passou a $45,4 \%$ na avaliação posterior por $\mathrm{HE}$, com um acréscimo de 10,4\% de casos com metástase. O estudo patológico por $\mathrm{HE}$ de todas as 85 pacientes mostrou 41 com LNS+, correspondendo a $48,2 \%$ do total, sendo 31 casos com macrometástase e 10 casos com micrometástase $(24,4 \%)$. No exame anatomopatológico dos linfonodos não-sentinelas ocorreu a presença de metástase em 10 casos, sendo que 2 foram casos de LNS falsamente negativo.

Caso a estatística do LNS seja realizada avaliando a proporção entre todos os casos, com e sem alteração patologica axilar, e os resultados do LNS (Tabela 3), tem-se $p<0,001$. Quando consideradas as duas amostras, de LNS e linfonodo, com os 35 pares discordantes, a estatística do $\chi^{2}$ de McNemar mostra $\mathrm{p}<0,001$.

Tabela 3 - Acurácia do exame histopatológico do linfonodo sentinela (LNS) em relação aos linfonodos axilares.

\begin{tabular}{|c|c|c|c|}
\hline \multicolumn{4}{|c|}{ Número de pacientes } \\
\hline & \multicolumn{2}{|c|}{ Linfadenectomia axilar (LNS e não-LNS) } & \multirow[t]{2}{*}{ Total } \\
\hline LNS & Positiva & Negativa & \\
\hline Positivo & 41 & 0 & 41 \\
\hline Negativo & 2 & 42 & 44 \\
\hline Total & 43 & 42 & $85 p<0,001$ \\
\hline
\end{tabular}

Os resultados das proporções binomiais realtivas ao mapeamento e ao status axilar são os seguintes: sensibilidade de 95,3\%, especificidade de $100 \%$ valor preditivo negativo de $95,5 \%$. A probabilidade pós-teste negativo é de $4,5 \%$.

Com os métodos associados e com o azul vital, temos, respectivamente, acurácia de $98,1 \mathrm{e}$ $96,8 \%$, com um LNS falsamente negativo em cada um dos métodos, sendo que a totalidade dos casos apresenta acurácia de 97,6\%.

\section{Discussão}

Na análise da técnica de localização do LNS, Nos et al. ${ }^{10}$ mostram-se estimulados com os resultados obtidos usando só o azul patente e, embora tenham-no identificado em $87,7 \%$ dos casos, a acurácia foi de $97,1 \%$.

Borgestein et al. ${ }^{11}$ referem que a biópsia do LNS, além de definir o estadiamento patológico da axila, tem baixa morbidade e custos menores tanto para a paciente quanto para o hospital. Utilizando linfocintilografia e rastreamento com o gama probe obteve identificação correta em 98,4\%, nos seus casos. O'Hea et al. ${ }^{12}$ usaram as três técnicas associadas e consideram que o uso de azul vital e a localização do radioisótopo com o probe são técnicas que se complementam, tendo conseguido localizar o LNS em 93\%, com acurácia de 95\%. Miltenburg et al. ${ }^{13}$, numa meta-análise incluíndo 11 estudos, com 912 casos, nos quais se usaram azul vital e radiofármaco, referem $84 \%$ de localizações e uma concordância de $98 \%$. Ressaltaran que, conforme a técnica usada e os critérios de inclusão, o LNS pode ser identificado em $97 \%$ dos casos.

Nossos dados validam o método para uma população similar a esta amostra, demonstrando que o LNS de fato reflete o status axilar ${ }^{1}$.

Os resultados da positividade do LNS mostram, quanto às variáveis idade e tamanho tumoral, a tendência da maior freqüência de positividade entre as mulheres mais jovens e tamanho tumoral maior que $2,0 \mathrm{~cm}$, conforme relato de outros autores ${ }^{4,6,9}$.

Quanto à associação de métodos e com azul vital observamos que a acurácia fica respectivamente em 98,1 e 96,8\% com um caso de LNS falsamente negativo para cada um dos métodos. Os nossos resultados estão de acordo com outros trabalhos, que validaram tanto o uso do ${ }^{99 \mathrm{~m}} \mathrm{Tc}$ quanto o corante azul ${ }^{10,14-16}$.

Gulec et al. ${ }^{17}$, usando apenas o detector gama probe $^{1}$, identificaram $94 \%$ dos LNS, sem nenhum falso-negativo, em 30 pacientes e concluíram que a biópsia do LNS tem validade clínica. Paganelli et al. ${ }^{18}$, realizando a linfografia, também com três soluções de tecnécio não filtradas e com partícu- 
las entre 50 e $1000 \mathrm{~nm}$, detectaram com o gama probe $97,6 \%$ dos LNS e a acurácia, na predição do status axilar, foi de 97,1\% (206 em 210 pacientes), referindo que é mais fácil a detecção com partículas maiores. Jaderborg et al. ${ }^{19}$ alertam que a técnica do LNS apresenta acurácia de $98,4 \%$, porém exige curva de aprendizado e que, ao usar a cirurgia do LNS, deve-se realizar termo de consentimento informado com a paciente.

Alguns autores discutem a importância do tamanho das partículas do colóide ligado ao tecnécio e a possibilidade de melhores resultados na detecção do LNS. Para Uren et $a .^{20}$, as partículas deveriam ter diâmetro entre 5-75 $\mathrm{nm}$. As partículas maiores do que $75 \mathrm{~nm}$, teriam entrada limitada nos linfáticos sob condições fisiológicas, porém isto é questionado por Gulec et al. ${ }^{17}$, que afirmam que o uso do filtrado de enxofre coloidal marcado com ${ }^{99 m}$ tecnécio (>75 a $220 \mathrm{~nm}$ ) ou solução coloidal não filtrada injetada ao redor do tumor primário não altera o resultado. É provável que as alterações patologicas próprias do linfonodo ou as decorrentes de outras doenças, ou lipossubstituição, possam afetar a captação do radiofármaco pelo $\mathrm{LNS}^{7,19}$. Tivemos três casos em que apenas parte do LNS estava azul e, à congelação, demonstravam importante lipossubstituição.

O ${ }^{99 m}$ tecnécio tem sido usado com várias soluções coloidais e as partículas apresentam tamanhos variados, com resultados igualmente satisfatórios. Dextran ligado ao tecnécio tem sido menos utilizado, porém os resultados são igualmente bons, não havendo consenso a respeito do tamanho ideal das partículas ${ }^{7-9}$.

$\mathrm{Na}$ literatura não encontramos a anotação de tempo e de valores de irradiação relacionados com o mapeamento do LNS. Para Brenner et al. ${ }^{21}$ a sensibilidade da cintilografia, com imagens obtidas em 1 a 2 horas, foi de $56 \%$, porém chegando a $86 \%$ com imagens mais tardias. Os nossos resultados demonstram que os grupos são distintos quanto às contagens gama e tempo decorrido desde o momento da injeção até o momento da detecção na cirurgia. Entretanto, fica estabelecido que a acurácia para a detecção do LNS nos dois grupos não difere. Os menores valores não impediram a detecção do LNS, porque o importante é a relação das contagens no LNS com as contagens de fundo no tecido em torno, que deve ficar pelo menos duas vezes menor. No caso da menor contagem, que foi 80 , a contagem de fundo oscilou entre 2 e 3 , sendo que este caso foi descoberto pelo corante e confirmado pelo detector manual, numa demonstração do valor da associação dos métodos.

Observou-se que, no transoperatório, em alguns casos ainda não detectados, o probe apresentava-se útil, orientando o ponto quente onde havia maior contagem e, então, mais facilmente encontrava-se o azul, confirmando a orientação. Estes casos habitualmente tinham LNS laterais ou no nivel II axilar. O azul vital foi encontrado em todos os casos, sendo que a abordagem cirúrgica era orientada pelo probe (local de maior contagem) ou pela conveniência do cirurgião na procura do ducto linfático corado nos casos em que se usou apenas o azul vital, com êxito.

Neste trabalho conseguimos definir que a linfocintilografia é útil, podendo mostrar mais de uma via até o nodo sentinela, e que as pacientes poderão receber a injeção do tecnécio, antes do procedimento cirúrgico ou com intervalo de até 17 horas, sem que se alterem os resultados corretos. Recomendamos, quando duas rotas forem mostradas na cintilografia, a procura de dois pontos com LNS. O mapeamento do LNS se mostrou factivel tanto com o radiofármaco quanto com o corante azul vital, mas os métodos associados mostraram $100 \%$ de resultados corretos. A acurácia do mapeamento ficou dentro do erro estatístico aceitável, o que nos permite sugerir que a linfadenectomia axilar necessita ser repensada, para os casos com axila negativa confirmados pela histopatologia do LNS.

\section{ABSTRACT}

Purpose: to evaluate the lymphoscintigraphy, the hand-held probe and the vital blue dye to identify the sentinel lymph node (SLN) in breast carcinoma and to establish sensitivity, negative predictive value (NPV) and overall accuracy of the SLN detection. Methods: eighty-eight consecutive attended patients, with clinically negative axillary lymph nodes were enrolled for this study using the technique of mapping with ${ }^{99 m}$ technetium dextran for scintigraphic images before surgery. In the operating room, five minutes before axillary incision, we injected $2 \mathrm{~mL}$ of $2.5 \%$ Bleu Patente V Sodique around the tumor and intradermally. Then the gamma probe helped to find out the hot spot where the SLN was supposed to be. The pathologic results of SLN were obtained by the standard technique with hematoxylin and eosin staining in seventy-seven patients.

Results: scintigraphy was conclusive for lymphatic basins in $62.1 \%$ of 58 cases, concerning SLN identification. In these conclusive patients, there were 9 in which 2 nodes appeared simultaneously without lymph vessel delineation. Using the gamma probe, at least one axillary SLN site could be found preoperatively in 45 cases (84.9\%), and intraoperatively, associated with blue dye, in all 53 patients. In 32 of 35 patients only vital blue dye was used with success. Concerning the groups "day" and "other day" (when biopsy was performed on the same day or the other day, after the radiolabeled injection), therefore different regarding time and gamma counts, both achieved the same results regarding SLN detection. Forty-one patients had positive axillary lymph nodes and only two had false negative SLN, giving sensitivity of $95.3 \%$, a NPV of $95.5 \%$ and overall accuracy of $97.7 \%$.

Conclusions: the lymph node mapping with the use of technetium and patent blue $V$, alone or associated, is feasible. The time since the injection of technetium up to surgery varied 
from 3 to $17 \mathrm{~h}$. The pathologic findings confirmed that the biopsied lymph node was the true sentinel node in $97.6 \%$ of the cases and validated the SLN mapping, and this should lead to an avoidance of complete axillary dissection as a routine procedure in patients with negative SLN.

KEY WORDS: Breast cancer. Lymphadenectomy. Axillary metastases. Sentinel lymph node.

\section{Agradecimentos}

Agradecemos aos colegas Sandra da C. Fuchs, Ana C. Bittelbrunn, José A. Cavalheiro, Rodrigo Cericatto e Miguel da Cunha Xavier, ao bioquimico Osmar de Oliveira, à enfermeira Eliane G. Rabin e à bolsista de iniciação científica Maria Fernanda Detanico,.

\section{Referências}

1. Krag DN, Weaver DL, Alex JC, Fairbank JT. Surgical resection and radiolocalization of the sentinel lymph node in breast cancer using a gamma probe. Surg Oncol 1993; 2:335-40.

2. Giuliano AE, Kirgan DM, Guenther JM, Morton DL. Lymphatic mapping and sentinel lymphadenectomy for breast cancer. Ann Surg 1994; 220:391-401.

3. Koller M, Barsuk D, Zippel D, Engelberg S, Ben-Ari G, Papa MZ. Sentinel lymph node involvement - a predictor for axillary node status with breast cancer has the time come? Eur J Surg Oncol 1998; 24:166-8.

4. Veronesi U, Paganelli G, Galimberti V, et al. Sentinelnode biopsy to avoid axillary dissection in breast cancer with clinically negative lymph-nodes. Lancet 1997; 349:1864 -7.

5. Morton D, Wen D-R, Cochran A. Management of earlystage melanoma by intraoperative lymphatic mapping and selective lymphadenectomy: a alternative to routine elective lymphadenectomy or watch and wait. Surg Oncol Clin North Am 1992; 1:247-59.

6. Rehman S, Sardi A, Spiegler E, Colandrea J, Frishberg D. Sentinel lymph node mapping for staging breast cancer: preliminary results of a prospective study. Md Med J 1999; 48:105-10.

7. Giuliano AE. Mapping a pathway for axillary staging: a personal perspective on the current status of sentinel lymph node dissection for breast cancer. Arch Surg 1999; 134:195-9.

8. Krasnow AZ, Hellman RS. Lymphoscintigraphy revisited: 1999. In: Freeman LM, editor. Nuclear Medicine Annual. $1^{\text {st }}$ ed. Philadelphia: Lippincott Williams \& Wilkins; 1999. p.17-55.
9. Noguchi M, Tsugawa K, Bando E, et al. Sentinel lymphadenectomy in breast cancer: identification of sentinel lymph node and detection of metastases. Breast Cancer Res Treat 1999; 53:97-104.

10.Nos C, Bourgeois D, Freneaux P, Zafrani B, Salmon RJ, Clough KB. Identification of sentinel lymph node in breast cancer: experience from the Institut Curie. Bull Cancer 1999; 86:580-4.

11.Borgestein PJ, Pijpers R, Comans EF, van Diest PJ, Boom RP, Meijer S. Sentinel lymph node biopsy in breast cancer: guidelines and pitfalls of lymphoscintigraphy and gamma probe detection. $\mathrm{J}$ Am Coll Surg 1998; 186:275-83.

12.O'Hea BJ, Hill AD, El-Shirbiny AM, et al. Sentinel lymph node biopsy in breast cancer: initial experience at Memorial Sloan-Kettering Cancer Center. J Am Coll Surg 1998; 186:423-7.

13. Miltenburg DM, Miller C, Karamlou TB, Brunicardi, FC. Meta-analysis of sentinel lympn node biopsy in breast cancer. J Surg Res 1999; 84:138-42.

14.Winchester DJ, Sener SF, Winchester DP, et al. Sentinel lymphadenectomy for breast cancer: experience with 180 consecutive patients: efficacy of filtered technetium 99m sulphur colloid with overnight migration time. J Am Coll Surg 1999; 188:597-603.

15. Miner TJ, Shriver CD, Jaques DP, ManiscalcoTheberge ME, Krag DN. Ultrasonographically guided injection improves localization of the radiolabeled sentinel lymph node in breast cancer. Ann Surg Oncol 1998; 5:315-21.

16.Reynolds C, Mick R, Donohue JH, et al. Sentinel lymph node biopsy metastasis: can axillary dissection be avoided in some patients with breast cancer? J Clin Oncol 1999; 17:1720- 6.

17.Gulec SA, Moffat FL, Carroll RG, et al. Sentinel lymph node localization in early breast cancer. J Nucl Med 1998; 39:1388-93.

18.Paganelli G, De Cicco C, Cremonesi M, et al. Optimized sentinel node scintigraphy in breast cancer. Q J Nucl Med 1998; 42:49-53.

19.Jaderborg JM, Harrison PB, Kiser JL, Maynard SL. The feasibility and accuracy of the sentinel lymph node biopsy for breast carcinoma. Am Surg 1999; 65:699-705.

20.Uren RF, Howman-Giles RB, Thompson JF. Regarding sentinel lymph node localization in early breast cancer. J Nucl Med 1999; 40:1403-6.

21.Brenner W, Ostertag $\mathrm{H}$, Czech $\mathrm{N}$, et al. Lymph scintigraphy and pre- and intraoperative gamma probe measurements for localization of sentinel lymph nodes (SLN) in breast cancer. Nuklearmedizin 2000; 39:82-7.

Recebido em: 2/8/2002 Aceito com modificações em: 23/9/2002

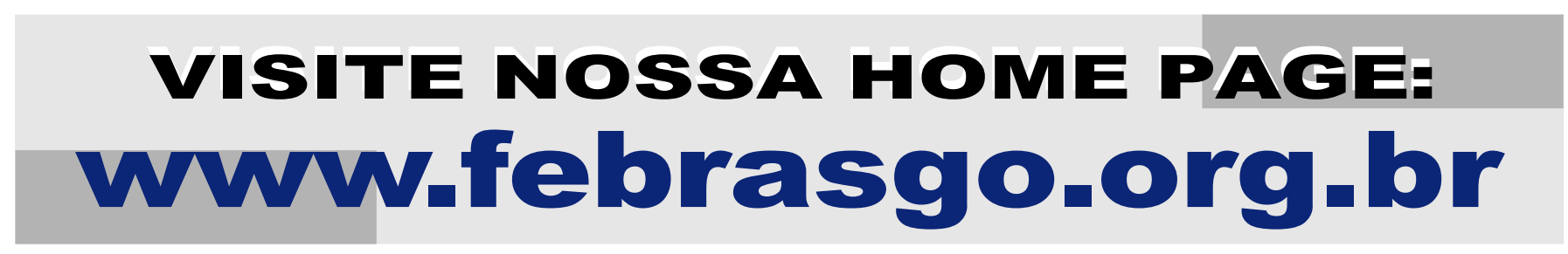

